\title{
Michel Foucault, dramaturge du vrai
}

\author{
Beatrice Barbalato $^{1}$
}

Foucault's work in the last years of his life revolved almost exclusively around the self (autos), the 'me, myself, and I'. According to Foucault, this concern for the self can be materialised only in the current, in calling upon the cura sui rather than the nosci te ipsum. Three stars form the 'Big Dipper' of his work: Kant's essay «What is Enlightenment?»; Socrates's last words as told in The Phaedo, when, just before dying, Socrates put the past, present, and future on the same footing; and the ancient practice of parrhesia (parrêsia), or speaking candidly in public. Foucault saw the cura sui as a necessarily ethical choice, a philosophy that postulated a genealogy of interruption, a construction of the subject beyond any telos (from the Greek $\tau \dot{\varepsilon} \lambda$ os meaning an end or objective). Foucault chose the philosophical principle of parrhesia to guide his behaviour. He read the first studies of Giuseppe Scarpat, a contemporary Hellenic scholar who analysed the full breadth of the term's original value.

Le travail des dernières années de Foucault s'est développé presque exclusivement autour de l'autos, le $m o i$, le je. Ce souci de soi ne peut se concrétiser selon Foucault que dans l'actuel, et en faisant appel à la cura sui plutôt qu'au nosci te ipsum. Dans la constellation de sa pensée, trois pôles forment en quelque sorte la Grande Ourse de son travail : l'essai de Kant «Qu'est-ce que les Lumières ? »; les derniers mots de Socrate rapportés dans le Phédon; et la pratique ancienne de la parrêsia, le franc-parler en public. La cura sui s'impose pour Foucault comme un choix éthique, une philosophie qui postule une généalogie de l'interruption, une construction du sujet au-delà de tout telos (du grec $\tau \hat{\lambda} \lambda_{0} \varsigma$, fin, objectif). Foucault a élu comme ligne de conduite le principe philosophique de la parrêsia. Il a lu les premières études de Giuseppe Scarpat, hélleniste contemporain, qui a analysé dans toute son ampleur la valeur originaire du terme.

\section{Entre historicisme et le hic et nunc, entre chronos et kairos}

In crescendo, la fonction du temps dans la construction du sujet devient presque une obsession pour Foucault. Ses derniers cours au Collège de France traitent de l'herméneutique du sujet et du

\footnotetext{
${ }^{1}$ Présidente de l'Observatoire de la mémoire autobiographique écrite, orale, iconographique. http://mediapoliseuropa.com/
} 
courage de la vérité. La pratique ancienne de la parrêsia va correspondre à cette idée d'une vérité non cachée et prononcée sur la place publique.

« La question dont je pars est 'Qu'est-ce que nous sommes, et qu'est-ce que nous sommes $a u$ jourd'hui ? Qu'est-ce que c'est que cet instant qui est le nôtre ?' »'.

La vérité vérifiable ne peut se réaliser qu'en assumant sa propre identité dans un devenir continuel $^{3}$ et dans une totale transparence en public. La philosophie des Cyniques, qui mettaient en avant l'idée d'une vie conduite dans toutes ses manifestations sur la place publique devient le point culminant, extrême et final de sa recherche.

Foucault a dédié une quantité considérable d'écrits à l'actualité. Lui-même a voulu être constamment présent sur les lieux des événements ${ }^{4}$. Être sur les lieux, pour Foucault, a signifié voir, constater, vérifier les conditions réelles. L'actualité pour lui s'identifiait avec la réalité, dans le sens que la langue anglaise donne au mot actually qui correspond à réellement (c'était d'ailleurs le sens du mot actuellement au XVIII' siècle : actuellement, c'est-à-dire : 'en acte').

Les dernières leçons de Foucault s'adressent à la pensée ancienne, aux techniques qui ont permis de faire un parcours d'édification de l'individu, au-delà de toute idée de transcendance. Foucault passe d'une «archéologie des sciences humaines »- c'est le sous-titre de Les mots et les choses - à la généalogie. Enfin, apparaît un tiers terme : attitude, qui reconduit tout comportement au présent, comme l'explique Judith Revel ${ }^{5}$. L'attitude est en effet une habilité, une disponibilité. Il ne s'agit jamais pour Foucault d'acquérir des convictions immuables mais de donner à son propre je l'attitude capable de se comporter dans le hic et nunc.

Harmoniser la cura sui et la pensée du dehors et vivre dans l'actualité sans retour possible - ni fuite en avant - deviennent des buts majeurs pour Foucault.

\footnotetext{
${ }^{2}$ Michel FOUCAULT, «Entretien de Michel Foucault avec André Berten », in id. Mal faire, dire vrai. Fonction de l'aveu en justice, Leçon du 22 avril 1981, édition établie par Fabienne BRION et Bernard E. HARCOURT, Presses universitaires de Louvain/ University of Chicago Press, 2012, p. 241.

${ }^{3}$ Cette idée est très proche de la critique que fait Nietzsche du Principium individuationis de Schopenhauer. Voir La Naissance de la tragédie, Die Geburt der Tragödie aus dem Geiste der Musik, 1872.

${ }^{4}$ Philippe ARTIÈRES, «Dire l'actualité », in Frédéric GROS dir., Foucault : le courage de la vérité, PUF, 2002, pp. 11-34.

5 Judith REVEL, « Promenades, petits excursus et régimes d'historicité », in Daniele LORENZINI, Ariane REVEL et Arianna SFORZINI dir., Michel Foucault : éthique et vérité 1980-1984, Paris, Librairie philosophique J. Vrin, 2013, p. 174.
} 


\section{Le présent et l'actuel. Le temps est dans l'espace}

Supprimer la frontière entre le dedans et le dehors, ainsi que l'idée d'un commencement comme point de départ originaire de notre pensée individuelle a été un des objectifs privilégiés des travaux de Foucault sur l'herméneutique du sujet. Dans ce souci de visibilité, la catégorie de l'espace gagne sur la diachronie. La quantité de marqueurs de situation que Foucault emploie dans ces œuvres est surprenante : avant, après, antérieure, a priori, de côté, archéologie dans l'espace général du savoir. Foucault semble opérer un développement horizontal dans lequel il étale les données. C'est presque une nécessité de sa part d'échelonner tout objet observé sur une surface. Foucault critique l'historicisme renfermé, finalisé, clos du XIX ${ }^{\mathrm{e}}$ siècle $^{6}$, pivot de notre culture actuelle.

« On aime à croire qu'à leur début les choses étaient en leur perfection, qu'elles sortirent éclatantes des mains du créateur, ou dans la lumière sans ombre du premier matin. L'origine est toujours avant la chute, avant le corps, avant le monde et le temps ; elle est du côté des dieux, et à la raconter on chante toujours une théogonie $»^{7}$.

Cette affirmation de Foucault extraite de son essai sur Friedrich Nietzsche manifeste une démarche qui s'oppose à l'idée de telos, de finalisation, de la reconnaissance d'un fil continu de l'histoire: une généalogie de l'interruption qui est capable de mettre à nu la discontinuité. Comme le dit aussi Castoriadis, l'origine ante res n'existe pas, l'origine est toujours ex post, elle est le résultat d'une narration et des institutions ${ }^{8}$.

Le travail de Foucault a constamment contrasté l'idée d'une pensée verticale, causale, totalisante avec celle de la discontinuité, du hasard.

Foucault affirme qu'avant l'essai de Kant Was ist Aufklärung? («Qu'est-ce que les Lumières ? » en 1784), aucun philosophe ne s'était posé la question «Qu'arrive-t-il aujourd'hui ? » - en allemand, de surcroît, Aufklärung signifie littéralement « élucidation, éclaircissement ». Au Siècle des Lumières, le thème de la contemporanéité s'affirme avec force, comme jamais auparavant. Il s'agit d'observer ce qui est disparate, interrompu, plutôt que ce qui sort de l'histoire " antiquaire », pour reprendre l'expression par laquelle Nietzsche définit l'histoire reconstruite généalogiquement en soumettant à un dessein toute diversité, tout écart. À la suite de Nietzsche, Foucault se demande: comment penser l'histoire de la vérité sans s'appuyer sur la vérité ?

\footnotetext{
${ }^{6}$ M. FOUCAULT, « Préface », in id. Les mots et les choses, Paris, Gallimard, 1966, p. 14.

${ }^{7}$ M. FOUCAULT, Texte 39, in Arnold I. DAVIDSON et Frédéric GROS dir., Philosophie-anthologie, Paris, Gallimard, 2004, p. 397. [Dits et écrits I, pp. 1004-1024].

${ }^{8}$ Cornelius CASTORIADIS, L'institution imaginaire de la société, Paris, Le Seuil, 1975, p. 218 et ss.
} 
Comment se défaire du «carnaval concerté » du généalogiste $?^{9}$ Comment défaire le déterminisme?

Foucault parle de l'essai de $\mathrm{Kant}^{10}$ à plusieurs reprises et il lui dédie une longue dissertation pendant une conférence à Berkeley en $1983^{11}$. Il souligne combien Kant se situe explicitement dans son actuel, en mettant l'individu au centre de l'argumentation ${ }^{12}$. Cet écrit se définit comme un type d'interrogation philosophique « qui problématise à la fois le rapport au présent, le mode d'être historique et la constitution de soi-même comme sujet autonome $»^{13}$. Loin des considérations sur le passé ou le futur, sur le déclin ou le progrès, Kant parle au présent du présent, dans lequel la raison et l'usage de la raison, mais aussi la nature et les sens ont un poids spécifique.

Pour Foucault, l'importance du texte de Kant consiste dans son questionnement: "Que sommes-nous actuellement ? $»^{14}$. Ce qui, aux yeux de Foucault, est le plus important est que Kant pose cette question dans un espace discursif présent sans tisser un rapport avec le passé et l'avenir, sans évoquer un avant et un après.

Comment, dans notre actualité, pouvons-nous rendre active cette pensée kantienne qui renonce à toute transcendance et à tout historicisme? Est-ce qu'on peut évoquer le vrai dans le présent sans faire recours au passé et à l'avenir?

\footnotetext{
${ }^{9}$ M. FOUCAULT, «Leçon sur Nietzsche. Comment penser l'histoire de la vérité avec Nietzsche sans s'appuyer sur la vérité », in id. Leçons sur la volonté de savoir. Cours au Collège de France 1970-1971, suivi du Savoir d'Edipe, édition établie sous la direction de François EWALD, Alessandro FONTANA et Daniel DEFERT, Paris, Gallimard/Seuil, 2011, p. 204.

${ }^{10}$ I. KANT, « Beantwortung der Frage: Was ist Aufklärung ? » in Berlinische Monatsschrift, 04 (Dezember), 1784, pp. 481-494. Emmanuel Kant Éléments métaphysiques de la doctrine du droit, trad. de l'allemand de Jules BARNI, Paris, éd. Auguste Durand, 1853 (pp. 281-288). https://fr.wikisource.org/wiki/Qu\%E2\%80\%99estce que les Lumi\%C3\%A8res \%3F

${ }^{11}$ M. FOUCAULT, « La culture de soi », in id. Qu'est-ce que la critique ? La culture de soi, éd. établie par Henri-Paul FRUCHAUD et Daniele LORENZINI, Introduction et apparat critique par D. LORENZINI et Arnold I. DAVIDSON, Paris, Librairie philosophique J. Vrin, 2015, Conférence à l'université de Californie à Berkeley du 12 avril 1983.

${ }^{12}$ Cf. : M. FOUCAULT, Dits et écrits IV, dir. Daniel DEFERT et François EWALD, en coll. avec Jacques LAGRANGE, Paris, Gallimard, 1994, pp. 562-578.

${ }^{13}$ M. FOUCAULT, «Le gouvernement de soi et des autres », in dir. A. I. DAVIDSON et F. GROS, Philosophieanthologie, op. cit., p. 871. [présent aussi dans Dits et écrits II, dir. D. DEFERT et F. EWALD, en coll. avec Jacques LAGRANGE, Paris, Gallimard, 1994, pp. 1381-1397 texte trad. de l'anglais]

${ }^{14}$ M. FOUCAULT, « La culture de soi », in id. Qu'est-ce que la critique, La culture de soi, op. cit., p. 84.
} 


\section{Dire vrai}

Malgré la conviction des Anciens que tout ce qui arrive est soumis au regard des Dieux, ils postulaient tout de même que les hommes, par leur propre cheminement, étaient en mesure de discerner le vrai et leur responsabilité propre.

Selon la conception archaïque grecque, seuls les « rois de justice » ${ }^{15}$, les poètes, les prêtres avaient accès à la Vérité. Ils voyaient le passé, le présent, le futur; la Vérité et la Mémoire n'étaient pas temporelles mais éternelles. Dans les poèmes homériques, l'aède pouvait voir dans les deux camps ; Hésiode utilise la première et troisième personne ${ }^{16}$. Ce regard omniscient était propre aux Muses ou aux Dieux. On est évidemment dans la pensée religieuse. On parle ici de la vérité, thème majeur de toute philosophie, par rapport aux sources que Foucault lui-même a évoquées dans ses écrits.

Foucault analyse comment, au début de notre civilisation, on a rendu visible et donc acceptable l'acte de rendre la justice. Il remonte à un des premiers récits que nous connaissons à ce propos: la compétition racontée au chant XXIII de l'Iliade. Pour les funérailles de Patrocle, Achille organise des jeux funèbres qu'il dote de nombreux prix. Diomède, Eumélos, Ménélas, Antilokhos, Mérion participent à la course de chars. Diomède, le plus valeureux, gagne le premier prix. Achille voudrait destiner le deuxième prix à Eumélos, position qu'il a manquée à cause d'Athéna qui a renversé son char; Antilokhos proteste parce que c'est lui qui est arrivé deuxième. C'est un fait, mais il l'a emporté grâce à une ruse, comme le dénonce Ménélas. Finalement, c'est à travers un franc-parler, un dire ouvert que Ménélas est désigné comme le meilleur des deux, mais Antilokhos aura quand même le second prix, grâce à la magnanimité de Ménélas qui, par l'aveu d'Antilokhos, s'est soucié seulement que la vérité soit connue. Ainsi, malgré toutes leurs stratégies pour conquérir leur propre indépendance, les résultats des coureurs vont correspondre, dans leur substance éthique, à la présentation qu'Achille avait faite des compétiteurs avant la course ${ }^{17}$. Ainsi, ce sont les Dieux qui ont créé des difficultés pour qu'à travers une visibilité évidente émerge la scénographie du vrai : «La course devrait avoir pour fonction

\footnotetext{
${ }^{15}$ «Il reste une tradition, poétique précisément, celle du 'roi de justice' tenant la 'balance', dispensateur et receveur tout à la foi du vrai et du faux. Parallèlement, toute vérité est énigme et tout diseur de vérité est lui-même énigme », Pierre VIDAL-NAQUET, « Préface », in Marcel DETIENNE, Les Maîtres de Vérité dans la Grèce archaïque, Paris, Livre de Poche, 2006, p. 45.

${ }^{16}$ M. DETIENNE, Les Maîtres de Vérité dans la Grèce archaïque, op. cit., p. 19.

${ }^{17}$ M. FOUCAULT, Mal faire, dire vrai. Fonction de l'aveu en justice, Leçon du 22 avril 1981, op. cit., p. 27.
} 
de manifester une vérité déjà reconnue. [...] la course ne doit rien être d'autre qu'une liturgie du vrai $»^{18}$. C'est - dit Foucault - le premier aveu judiciaire de notre culture où l'énonciation de la vérité se structure d'emblée comme un tribunal. Cet épisode souligne combien, malgré l'influence des Dieux, il a été retenu nécessaire que la vérité soit sollicitée, provoquée, manifestée, partagée : même si elle a été guidée par le haut, elle a besoin d'être reconnue, justifiée, racontée à un moment temporel précis. Pour Edipe, l'occasion venue est celle de la péripétie : à un moment culminant, toutes les connaissances sur sa vie se retournent dans leur contraire (cf. la Poétique d'Aristote), et c'est là que la vérité se dévoile. Dans la culture grecque où l'ambiguïté et l'énigme jouent un rôle essentiel, la vérité est cachée puis découverte. À côté d'une vérité absolue, divine, les hommes doivent la chercher, la comprendre. Rien ne leur est donné sans recherche. Tout est prédisposé pour faire coller l'agir des hommes à la volonté des Dieux. Les mots aussi ont une grande importance : agôn, comme le rappelle Pierre Vidal-Naquet à propos de la compétition en l'honneur de Patrocle, désigne à la fois l'assemblée et le combat ${ }^{19}$. Au début de notre civilisation ${ }^{20}$, un glissement de signification pouvait s'opérer d'un mot à l'autre.

Dans son livre Les Maîtres de Vérité dans la Grèce archaïque, Marcel Detienne montre comment, dans des inscriptions trouvées sur des tablettes en os datés de 500 ans av. J. C., le mot Alètheia (Vérité) était accompagné d'autres mots qui en contredisaient ou en amplifiaient la signification $^{21}$. Dans d'autres sources, Alètheia ${ }^{22}$ est associé à Apatè, tromperie. Ce parcours au

\footnotetext{
${ }^{18}$ Ibid., p. 28.

${ }^{19}$ P. VIDAL-NAQUET, «Préface », in M. DETIENNE, Les Maîtres de la Vérité dans la Grèce archaïque, op. cit., p. 49.

${ }^{20}$ On se rappelle l'Orestie, la trilogie de tragédies d'Eschyle (Agamemnon, les Choéphores et les Euménides), une œuvre où le premier tribunal humain se réalise par la volonté d'Athéna, et où les Erinyes sont transformées en Euménides.

${ }^{21}$ M. DETIENNE, Les Maîtres de Vérité dans la Grèce archä̈que, op. cit., p. 24.

${ }^{22}$ Heidegger n'est évoqué que rarement par Foucault. Et pourtant on rappelle ici même si c'est de manière latérale, que sur le mot alètheia Heidegger a mené d'importantes réflexions. Cette parole - autour de laquelle selon Heidegger se sont produits des équivoques - ne peut pas simplement signifier le dévoilement d'une vérité vue comme un objet à saisir, en d'autres termes comme le résultat pur et simple d'une procédure de vérification. Pour Heidegger, on a perdu au fil du temps le sens primordial de la vérité comme un processus de recherche, pour l'isoler comme un datum en soi et pour soi. Cf. Martin HEIDEGGER, De l'essence de la vérité. Approche de l'«allégorie de la caverne» et du «Théétète» de Platon, texte établi par Hermann MÖRCHEN, trad. de l'allemand par Alain BOUTOT, Paris, Gallimard, 2001 [1931 -1932].

${ }^{22}$ Un mot qui se différencie du concept de véridicité (c'est-à-dire, ce qui correspond au vrai, comme une donnée objective. La véridification porte sur le processus et ses circonstances du comment, quand, et où dire le vrai).
} 
long de plusieurs siècles témoigne de la recherche d'une mémoire laïque ${ }^{23}$, qui s'oppose à la parole magico-religieuse et transcendantale.

Le chemin a été long pour saisir une vérité plus expérimentée, pour s'affranchir de cette dominante surnaturelle. L'idée de tribunal, d'aveu survient pour rendre visible un processus de véridification, qui arrive à surmonter le pouvoir des Dieux et celui d'Édipe qui échappent tous les deux à une visibilité manifeste :

quand la Grèce classique apparait - Sophocle en représente la date initiale, le point d'éclosion - , ce qui doit disparaître pour que cette société existe, c'est l'union du pouvoir et du savoir. À partir de ce moment, l'homme du pouvoir sera l'homme de l'ignorance. Finalement, ce qui est arrivé à CEdipe c'est que, pour savoir trop, il ne savait rien. Pas parce qu'il pouvait trop. À partir de ce moment, Edipe va fonctionner comme l'homme du pouvoir, aveugle, qui ne savait pas, et qui ne savait parce que il pouvait trop ${ }^{24}$.

Lorsque Foucault analyse la tragédie $E$ dipe roi de Sophocle, ce qui est décisif pour la découverte du vrai, ce n'est pas la vérité d'Apollon et de Tirésias (l'un qui voit tout et l'autre qui est aveugle). Ils en sont même les garants. C'est le cœur qui n'accepte pas une vérité qui fait abstraction des preuves.

Ces preuves qui porteront à la vérité ne sont pas données non plus par Edipe et Jocaste : ils s'interrogent et reconstruisent les événements et ne trouvent rien qui puisse les faire responsables des crimes. Ils se doutent aussi du pouvoir de prédiction des dieux et nourrissent l'illusion «que l'on peut échapper au destin et que la technique humaine permet de passer à travers les trames et les fils qui ont été tendus par les dieux $»^{25}$.

Edipe dispose d'une tekhnê, un terme qui apparaît trois fois dans le texte de Sophocle : il possède l'art de gouverner, de guérir, de naviguer. La véridiction d'EEdipe est condamnée par le chœur au motif qu'en partie cette tekhnê a produit des excès tyranniques. Le troisième degré de véridiction se réalise avec l'interrogatoire des deux serviteurs : le messager qui vient annoncer que Polybe est mort, et le berger qui devait tuer CEdipe enfant et qui, en contrevenant aux ordres,

${ }^{23}$ Cette mémoire laïque s'est instaurée surtout grâce aux militaires et à la réforme des hoplites. Les militaires étaient organisés sur la base de classes d'âge et groupes de fratries indépendamment de tout autre facteur. $C f$. M. DETIENNE, «Les procès de laïcisation », in id. Les Maîtres de Vérité dans la Grèce archä̈que, op. cit., pp. 153183.

${ }^{24}$ M. FOUCAULT, Dits et écrits II, op. cit., p. 569.

${ }^{25}$ M. FOUCAULT, Mal faire, dire vrai. Fonction de l'aveu en justice, op. cit., p. 61. 
l'a libéré. Le berger est obligé de dire la vérité, sous la menace de la torture. Il y a une confrontation judiciaire entre les deux témoins ; encore une fois, émerge la nécessité d'un tribunal pour manifester l'évidence de la vérité. Le messager de Corinthe demande au berger qui lui a donné l'enfant, et le berger répond : « Oui, moi-même je l'ai trouvé dans le val de Cithéron, cet enfant. Oui, je gardais les troupeaux. Oui, c'est moi qui dégageai tes deux pieds [...]. Oui, c'est à moi que Jocaste t'avait remis [...] C'est moi qui ne l'ai pas tué - autos ${ }^{26}$. Cette vérité est incontestable et le chœur l'accepte. Ce n'est pas la prévoyance des dieux qui établit la vérité, ni le roi et leurs enquêtes qui, déductivement, procèdent par exclusion, mais la parole directe, prononcée par un moi, qui n'exploite pas une prérogative a priori - celle d'un dieu, d'un devin, d'un roi et qui, d'une manière circonstanciée dans le temps et dans l'espace - qu'il s'agisse des faits ou du témoignage sur ceux-ci -, permet de mettre en place un processus de véridiction. Ces témoignages de deux serviteurs ne contrastent pas avec la prophétie des Dieux, qu'CEdipe avait mise en cause. Avec (Edipe, une théâtralisation, une mise en scène du témoignage prennent forme : une forme primordiale de parrêsia ${ }^{27}$, d'un franc-parler comme assomption visible à tous des responsabilités à la première personne. On reviendra plus loin sur ce terme.

\section{La mémoire laïque}

Foucault s'est donc intéressé au statu nascenti du tribunal comme mise en scène du moment où un verdict est prononçable et prononcé dans l'actuel. Où se situe la vérité ? Comment devient-elle visible ? Quel est son centre gravitationnel ? On sait que l'ambiguïté a été la colonne vertébrale de la culture grecque ${ }^{28}$. Au temps des Sophistes, elle devient le centre même de la pensée, jusqu'aux excès que Platon ridiculise en disant que ces discours peuvent signifier en même temps une chose et son contraire ${ }^{29}$.

En fait, la parole devient pur instrument d'une technique de persuasion. Pour les Sophistes, la doxa, l'opinion, peut traiter de tous les arguments, peut dire et contredire. La vérité/Vérité de-

\footnotetext{
${ }^{26}$ Ibid., p. 69.

${ }^{27}$ J'écris parrêsia lorsque je me réfère à l'œuvre de Foucault et parrhesia quand je mentionne les textes de Scarpat.

${ }_{28}^{28}$ M. DETIENNE, «Les procès de laïcisation », in id. Les Maîtres de Vérité dans la Grèce archaïque, op. cit., pp. 196-197.

${ }^{29}$ Ibidem. Il se réfère à la République de Platon.
} 
vient objet du discours, de la rhétorique. Peut-on se soustraire à ce piège ? Foucault avance l'idée que l'éthos du locuteur devrait en être le garant.

A-lètheia signifie littéralement dé-celer, voir clair, ôter le voile. Selon Detienne, toute la recherche de la connaissance et de la vérité se développe autour du mot esti, être, qui, comme le remarque Benveniste, n'est nullement une nécessité dans toutes les langues ${ }^{30}$.

Se référant à la table des dix catégories linguistiques établies par Aristote, Benveniste affirme : «La langue fournit la configuration fondamentale des propriétés reconnues par l'esprit aux choses. [...] Au-delà des termes aristotéliciens, au-dessus de cette catégorisation, se déploie la notion d"être' qui enveloppe tout $»$.

Pour Foucault, on devrait apprendre à être dans la visibilité, se soucier de l'apparaître autant que de l'être, et assumer cette posture :

Le rôle de la philosophie n'est pas de découvrir ce qui est caché, mais de rendre visible ce qui précisément est visible, c'est-à-dire de faire apparaître ce qui est si proche, ce qui est si immédiat, ce qui est si intimement lié à nous-mêmes qu'à cause de cela nous ne le voyons pas. Alors que le rôle de la science est de faire connaitre ce que nous ne voyons pas, le rôle de la philosophie est de faire connaître ce que nous voyons ${ }^{32}$.

Dans l'antiquité, c'est dans le domaine militaire qu'émerge une conception du partage qui n'a rien à faire avec le pouvoir religieux. Tout est joué au milieu, es meson : la répartition du butin, toute confrontation, etc. "Quand Polybe », rappelle Detienne, "veut parler du privilège de parole des guerriers macédoniens, il parle de leur égalité de verbe, de leur isègoria $»^{33}$, un mot qui a une grande affinité avec isokratia. L'histoire d'une mémoire laïque s'est développée en parallèle à la pensée magico-religieuse, en constituant les bases d'une pensée démocratique. Sa prérogative a été d'être manifeste, de jouer dans l'espace visible, de ne pas s'inscrire dans l'idée d'une verticalité de la connaissance mais au centre d'un processus partagé.

${ }^{30}$ M. DETIENNE, Les Maîtres de Vérité dans la Grèce archaïque, op. cit., pp. 231-233.

${ }^{31}$ Émile BENVENISTE, Problèmes de linguistique générale, v. I, Paris, Gallimard, 1966, p. 70. Voir aussi les pages 71 et ss.

${ }^{32}$ M. FOUCAULT, «La philosophie analytique de la politique », in id. Daniel DEFERT et François EWALD, dir., avec la collaboration de Jacques LAGRANGE, Dits et écrits III, Paris, Gallimard, 2001, pp. 540-541. [1994].

${ }^{33}$ M. DETIENNE, Les Maîtres de Vérité dans la Grèce archaïque, ch. «Le procès de laïcisation », op. cit., p. 168 et 171 . 
Le visible et l'invisible sont un thème cher à Merleau-Ponty. Des liens entre Foucault et Merleau-Ponty ont été mis en lumière par Judith Revel ${ }^{34}$. Loin de l'idéalisme et de l'empirisme, Merleau-Ponty écrit : « Notre lien natal avec le monde, elle [la philosophie] ne pense pouvoir le comprendre qu'en le défaisant pour le refaire, qu'en le constituant, en le fabricant. [...] Il est donc essentiel à la philosophie réflexive de nous replacer, en-deçà de notre situation de fait, à un centre des choses, d'où nous procédions, mais par rapport auquel nous étions décentrés ${ }^{35}$. Dans ses « Notes de travail », Claude Lefort évoque Kafka : «Kafka disait déjà que les choses se présentaient à lui, 'non par leurs racines, mais par un point quelconque situé vers leur milieu'. Il le disait pour témoigner de sa misère, sans doute; mais le philosophe qui se déprend du mythe de la racine accepte résolument de se situer en ce milieu et de partir de 'ce point quelconque' $»^{36}$.

Raisonner en étant au centre, développer une argumentation en se situant au milieu, se positionner au centre comme région médiane $e^{37}$, signifie nier la généalogie.

\section{Un regard rapproché, chirurgical, calligrammatique}

Être au centre signifie pour Foucault assumer une morale antistratégique : «Il faut tout à la fois guetter, un peu au-dessous de l'histoire, ce qui la rompt et l'agite, et veiller un peu en arrière de la politique sur ce qui doit inconditionnellement la limiter $»^{38}$. C'est la position d'un regard rapproché, le regard d'un anatomiste, de celui qui utilise l'écriture comme un bistouri : une écriture qu'il faudrait étudier à fond, parce que Foucault, écrit Philippe Artières, « ne cite pas, il détache, découpe des énoncés» ${ }^{39}$, «Il repère les organes, suit les cassures, les brisures $»^{40}$.

\footnotetext{
${ }^{34}$ Cf. : J. REVEL, Foucault avec Merleau-Ponty, Paris, Vrin, 2015.

${ }^{35}$ Maurice MERLEAU-PONTY, Le visible et l'invisible, suivi de "Notes de travail », texte établi par Claude LEFORT, Paris, Gallimard, 1964, p. 53. La réflexion de Merleau-Ponty configure le philosophe comme quelqu'un qui en partant de l'extérieur va vers le centre des choses.

${ }^{36}$ Claude LEFORT, « Notes de travail », in M. MERLEAU-PONTY, Le visible et l'invisible, op. cit, pp. 351-352. Réf. Franz KAFKA, Journal intime, trad. et intr. de Pierre KLOSSOWSKI, Paris, Grasset, 1945.

${ }^{37}$ M. FOUCAULT, « Préface », in id. Les mots et les choses, op. cit., p. 12

${ }^{38}$ M. FOUCAULT, «Inutile de se soulever? », in id. Dits et écrits III, op. cit., p. 794.

${ }^{39}$ Philippe ARTIÈRES «Dire l'actualité » in Frédéric GROS dir., Foucault : le courage de la vérité, P.U.F., 2002, p. 26.

${ }^{40}$ Ibid., p. 27.
} 
«On pourrait aussi citer Plutarque », écrit Foucault, « disant que médecine et philosophie ont, ou, plus exactement sont mia khôra (une seule région, un seul pays) ${ }^{41}$. Il remarque par ailleurs : «Épictète ne voulait pas que son école soit considérée simplement comme une école ou un lieu de de formation. Il voulait plutôt que son école soit considérée comme un cabinet médical, ce qu'il appelait un iatreion [hôpital] $»^{42}$.

Philon d'Alexandrie utilise à propos de la parrhesia des mots comme cicatrice, cicatriser ${ }^{43}$. Foucault, dans L'herméneutique du sujet, rappelle que des locutions comme " ouvrir les abcès ", «nettoyer les plaies », etc., formulaient des indications très concrètes de la cura sui.

Foucault revient à maintes reprises sur l'idée que la cura sui soit déployée avec des techniques claires et dans un moment spécifique et nécessaire comme dans des interventions chirurgicales. Quand il parle de De tranquillitate animi, lorsque Sérénus s'adresse à Sénèque, son directeur de conscience, pour examiner s'il est maître de ses actions, il énumère sic et simpliciter (expositio animae) ce qui lui plaît et ce qui ne lui plaît pas. Ni les questions ni les réponses ne cherchent à découvrir des désirs cachés, des maladies secrètes.

C'est principalement à travers la langue qu'on peut poursuivre ces propos. Elle avait fait déjà l'objet de multiples considérations foucaldiennes, notamment dans Les mots et les choses ${ }^{44}$ où, tout au début, Foucault mentionne Borges évoquant une certaine encyclopédie chinoise (Borges, Otras inquisiciones, 1952) dans laquelle les animaux sont classés selon la contingence de leur condition (animaux embaumés, apprivoisés, etc.), ou selon leurs propriétés, etc. : impossible d'encoder le monde dans toutes ses singularités, dans tous ses états, de le nommer sur la base d'une réalité additionnelle. Entre les mots et les choses, chaque période a établi un ordre. Il est certain qu'après l'Âge classique, le rapport de cohérence entre la théorie de la représentation et celle du langage, des ordres naturels, de la richesse et de la valeur a profondément changé. Dans le chapitre "Les suivantes », il évoque Las meninas de Velasquez, tableau où les significations se renvoient les unes aux autres en se reflétant et s'enrichissent à l'intérieur du tableau qui devient en soi et pour soi un univers de [auto]références. Tout y est. Le tableau est un champ signifiant, un cercle indépendant de tout extérieur, à l'intérieur duquel Velasquez même, en tant qu'auteur, se positionne.

\footnotetext{
${ }^{41}$ M. FOUCAULT, L’herméneutique du sujet, Cours au Collège de France 1981-1982, édition établie sous la direction de François EWALD et Alessandro FONTANA, et par F. GROS, Paris, Gallimard-Seuil, 2001, 94.

${ }^{42}$ M. FOUCAULT, «La culture de soi », in id. Qu'est-ce que la critique? La culture de soi, op cit., p. 94.

${ }^{43}$ Giuseppe SCARPAT, Parrhesia greca, parrhesia cristiana, Brescia, Paideia edizioni, 2001, p. 69.

${ }^{44}$ M. FOUCAULT, «Préface », in id. Les mots et les choses, op. cit.. L'italique est dans le texte de Foucault.
} 
Dans cette logique, le calligramme devient pour Foucault la forme d'expression la plus indépendante, autonome, autoréférentielle.

Quel est, donc, le rapport idéal entre les mots et les choses ? Foucault définit la première version du dessin Ceci n'est pas une pipe (1926), de Magritte, comme un 'calligramme' : une forme d'expression tautologique et antirhétorique. La ressemblance n'est pas la chose : «Peinture du 'Même', libérée du 'comme si' »". Le calligramme est une tautologie dans le sens où il renvoie à lui-même. À l'opposé de la rhétorique, qui peut dire deux fois la même chose avec des mots différents, le calligramme efface la base de notre civilisation linguistique, celle de montrer et nommer, imiter et signifier ${ }^{46}$. Foucault délimite des champs d'observation de plus en plus restreints, circonscrits, circulaires.

Le langage n'est pas un révélateur, il est arbitraire. Le sujet ne doit pas poursuivre le nosce te ipse (qui reste mystérieux) mais la cura sui, qui se réalise comme et dans un microcosme.

Comme le dit aussi Nietzsche :

J'estime un philosophe dans la mesure où il est en état de donner un exemple. [...] l'exemple doit être donné par la vie visible et non pas seulement par les livres; il doit donc être donné, comme l'enseignaient les philosophes de la Grèce, par l'expression du visage, l'attitude, le vêtement, le régime alimentaire, les mœurs, plus encore que par les paroles et surtout que par l'écriture ${ }^{47}$.

Ainsi, pour Foucault comme pour Nietzsche, chaque geste, action, réflexion doit être factualisé dans une actualité choisie, circonscrite, exemplaire. Être au milieu comme suspendu, coupé de toute influence prédéterminée, pour travailler sur soi, pour essayer de définir un comportement exemplaire et de s'y reconnaitre.

Foucault traite cet argument dans ses deux derniers cours L'herméneutique du sujet (cours au Collège de France, 1981-1982), et Le courage de la vérité (1984). Il y privilégie la cura sui plutôt que le nosce te ipsum. On a supposé que cette notion de cura sui n'a pas été transmise à cause de la désagrégation du monde ancien. Peut-être a-t-on plutôt privilégié l'idée de la con-

${ }^{45}$ M. FOUCAULT, Ceci n'est pas une pipe, Saint Clément de Rivière, Fata Morgana, 1973, p. 41

${ }^{46}$ Ibid., pp. 15-16.

${ }^{47}$ F. NIETZSCHE, « Schopenhauer éducateur », in id. Considérations inactuelles, textes et variantes établis par Giorgio COLLI et Mazzino MONTINARI, v. III et v. IV, traduit de l'allemand par Henri-Alexis BAATSCH, Pascal DAVID, Cornélius HEIM, Philippe LACOUE-LABARTHE et Jean-Luc NANCY, Paris, Gallimard, 1990, p. 29. [1874]. 
naissance théorique et rationnelle, selon la dictée d'Aristote (Métaphysique 1,1) : "Tous les hommes aspirent à la connaissance » - un principe fascinant, et qui réunit en soi désir et connaissance ${ }^{48}$.

La cura sui consisterait dans un engagement constant et continu d'une vie, une vie qui n'a pas de destin, ni de destination, où cette idée de chronologie qui induit à la conception d'une existence à terme ne compte pas, et où l'âge n'a pas d'importance. Lucien rappelle que Licinius s'adresse à Hermotime, qui a soixante ans ${ }^{49}$. Foucault cite la lettre d'Épicure à Ménécée où il affirme qu'il n'est jamais trop tard pour s'occuper de sa propre âme ; lorsqu'on est jeune, il faut philosopher pour avoir la fermeté d'un vieux ; et un homme vieux, lui, doit philosopher pour rajeunir :

«C'est-à-dire, c'est retourner le temps, ou en tout cas s'arracher au temps $»^{50}$.

Cet actuel se réalise aussi dans la stricte alliance entre écouter, écrire et transcrire ses propres réflexions. Sénèque recommandait d'alterner lecture et écriture ${ }^{51}$ qui étaient considérés comme le cœur même d'une subjectivité toujours poursuivie dans l'actualité. Cette alternance établit un lieu de croisement, de production de sens ; Épictète dit: « Puisse la mort me saisir en train de penser, d'écrire et de lire ces phrases-là $)^{52}$, Arrien transcrit les paroles d'Épictète fidèlement et simultanément à leur énonciation. Il y a, dit Foucault, un corps du langage, une matérialité, une immédiateté (in mediatus, sans interposition) qui n'a rien de rhétorique, c'est-à-dire de feint, d'artificiel, de séduisant ${ }^{53}$. Cette idée d'une mémoire inscrite de façon permanente dans l'actualité était déjà une réalité dans la poésie orale, prononcée par cœur des aèdes.

L'aède et le sage gardaient et transmettaient la mnemê, la mémoire (et ils participaient directement à cette mnemê) - on pouvait les écouter dans l'actualité du récit. Et pas seulement :

Dans l'idée qu'il faut avoir les logoi (les logoi boêthikoi, le logos de secours) sous la main, vous voyez que c'est un peu quelque chose d'autre que cette préservation de l'éclat de la vérité dans la mémoire de ceux qui participent à la mnemê. [...] Il faut l'avoir [la mnemê] sous la main, c'est-à-dire qu'il faut l'avoir en quelque sorte presque dans les muscles. Il faut l'avoir de telle manière que l'on puisse la [la paraskeuê] réactualiser immédiatement et sans délai, de

\footnotetext{
${ }^{48}$ M. FOUCAULT, Leçons sur la volonté de savoir, op. cit., p. 19.

${ }^{49}$ M. FOUCAULT, L'herméneutique du sujet, Cours au Collège de France 1981-1982, op. cit., p. 89.

${ }^{50}$ Ibid., p. 85.

${ }^{51}$ Ibid., p. 341. Lettre 84

${ }^{52}$ Ibid., p. 342. (Épictète, Entretiens, III, 5,11, p. 23).

${ }^{53}$ Ibid., p. 350.
} 
façon automatique. Il faut que ce soit en réalité une mémoire d'activité, une mémoire d'acte beaucoup plus qu'une mémoire de chant ${ }^{54}$.

Foucault souligne encore qu'Épictète donnait une grande importance à l'éducation des représentations à tout âge, pour entraîner l'attitude à les surveiller ${ }^{55}$.

\section{Le courage de la vérité}

Cette année, je voudrais continuer l'étude du franc-parler, de la parrêsia comme modalité du dire-vrai. [...] L'alèthurgie serait, étymologiquement, la production de la vérité, l'acte par lequel la vérité se manifeste. Donc laissons de côté les analyses de type 'structure épistémologique' et analysons un peu les 'formes alèthurgiques ${ }^{56}$.

[...] la plupart du temps, les gens croient que ce qu'on doit faire, c'est dévoiler, libérer, déterrer la réalité cachée du soi. Mais le soi, je crois, ne doit pas être considéré comme une réalité qui peut être cachée; je crois que le soi doit être considéré comme le corrélatif de technologies développées à travers notre histoire. Le problème, alors, ce n'est pas de délivrer, ce n'est pas de 'libérer' le soi, mais d'envisager comment il serait possible d'élaborer de nouveaux types, de nouvelles sortes de relations à nous-mêmes ${ }^{57}$.

La vérité pour Foucault n'a donc pas de caractère salvifique, ni ne doit être révélée. Elle n'est pas non plus une entité qui vit en-dehors du sujet : elle n'est ni un achèvement ni une récompense, dit Foucault ${ }^{58}$, elle peut être identifiée dans l'engagement continu de poursuivre la cura sui, et d'essayer de faire correspondre intime et ethos. Foucault rappelle le Faust de Lessing, dont subsistent quelques fragments ${ }^{59}$. Ce n'est pas la connaissance interprétée comme un absolu qui sauve Faust : son drame traverse le conflit de la conversion du savoir spirituel en savoir de connaissance, qui est, au XVII ${ }^{\mathrm{e}}$ siècle, au centre des raisonnements de Descartes, Pascal, Spino-

\footnotetext{
${ }^{54}$ Ibid., p. 311.

${ }^{55}$ Ibid., p. 136.

${ }^{56}$ M. FOUCAUT, Le courage de la vérité. Le gouvernement de soi et des autres, v. II, Paris, Gallimard-Seuil, 2009, pp. 4-5.

${ }^{57}$ M. FOUCAULT, « La culture de soi », in id. Qu'est-ce que la critique ? La culture de soi, op. cit., p. 98.

${ }^{58}$ M. FOUCAULT, L'Herméneutique du sujet, op. cit., p. 20.

${ }^{59} \mathrm{http} / / /$ guide.supereva.it/bibliofilia/interventi/2010/02/faust-tra-mito-e-leggenda.
} 
$\mathrm{za}^{60}$. On est au seuil du Siècle des Lumières, période étudiée par Starobinski justement à propos de la subjectivité. Il met en parallèle les idées de Rousseau (qui, avec les Confessions, donne une place privilégiée à l'intime) et de Diderot. Le titre Diderot, un diable de ramage ${ }^{61}$, joue avec les mots ramage comme ramification et Rameau, le musicien - Le Neveu de Rameau étant une œuvre de Diderot. Entre le moi du philosophe et le lui, le neveu du célèbre musicien Rameau, s'instaure une relation dialogique visant à extérioriser, à désocculter ce qui est interne ${ }^{62}-$ à faire correspondre le dehors au dedans, sans zones intermédiaires.

Starobinski souligne combien Diderot a eu le courage de se mettre en discussion, de mettre ses convictions à l'épreuve.

Ce discours est bien celui de Foucault, grand anatomiste des techniques qui soutiennent le courage de dire vrai et d'opérer une rupture avec une certaine idée du présent - c'est-à-dire ce qui émerge avant nous, en lui opposant le concept d'actualité ${ }^{63}$. Qu'est-ce que l'actuel ? C'est privilégier l'acte et non le fait. Foucault, en se référant à Marc-Aurèle, parle de parastêma, qui n'est pas un précepte, mais quelque chose que nous devons avoir toujours dans l'esprit et sous les yeux d'une manière concrète. Et «la seule instance de réalité qui existe pour le sujet, c'est l'instant lui-même : l'instant infiniment petit qui constitue le présent, avant lequel rien n'existe plus et après lequel tout est encore incertain $»^{64}$. Le présent est ici conçu non comme une entité qui est devant nous mais comme l'instant instantané : « Et par 'pensée' je n'entends pas exclusivement la philosophie, ni la pensée théorique, ni la connaissance scientifique ; je ne veux pas analyser ce que les gens pensent par opposition à ce qu'ils font, mais ce qu'ils pensent quand ils font ce qu'ils font ${ }^{65}$.

\footnotetext{
${ }^{60}$ M. FOUCAULT, L'Herméneutique du sujet, op. cit., p. 296.

${ }^{61}$ J. STAROBINSKI, Diderot, un diable de ramage, Paris, Gallimard, 2012. Les études présentées dans ce texte remontent aux années 1970-1990, et précèdent, donc, celles sur Rousseau.

On peut écouter sur ce thème une interview de Didier LAGARDE avec Starobinski :

http://www.franceculture.fr/emission-repliques-1-art-de-lire-2013-01-26.

${ }^{62}$ Ibid., pp. 117-118. Voir le Ch. «L'incipit du 'Neveu de Rameau'».

${ }^{63}$ J. REVEL, " Promenades, petits excursus et régime d'historicité », in Daniele LORENZINI, Ariane REVEL et Arianna SFORZINI dir., Michel Foucault : éthique et vérité 1980-1984, op. cit., p. 174.

${ }^{64}$ M. FOUCAULT, L'Herméneutique du sujet, op. cit., p. 279. C'est notamment un thème qui traverse l'Être et le temps d'Heidegger.

${ }^{65}$ M. FOUCAULT, « La culture de soi », in id. Qu'est-ce que la critique? La culture de soi, op. cit., p. 85.
} 


\section{Le rapport entre vérité de soi et oubli de soi}

Sur ce thème de l'actuel, d'une mémoire vivante jamais classée ni fossilisée, Foucault ne pouvait pas ne pas rencontrer la pensée de Socrate. L'importance de ne pas permettre d'être obligé à se démentir (à s'oublier), la décision, par contre, de s'oublier une fois venu le moment où ses convictions sont partagées, sont les argumentations socratiques qu'on trouve dans les Dialogues de Platon.

Dans le dernier cours prononcé par Foucault au Collège de France, entre février et mars 1984, quelques mois avant sa disparition, la mort de Socrate, son rapport à la parresîa, au franc-parler, prennent une signification prégnante. Foucault écrit : «Ce qui traverse, me semble-t-il, tout le cycle de la mort socratique, c'est bien l'établissement, la fondation, dans sa spécificité non politique, d'une forme de discours qui a pour préoccupation, qui a pour souci le souci de soi ${ }^{66}$.

Ailleurs, il précise : «c'est du rapport entre vérité de soi et oubli de soi qu'il va être question tout au long de ce cycle $»^{67}$. Nous voilà pris par la main et conduits par Foucault au fil de son ultime vision du temps.

Socrate est vigilant : il ne veut pas risquer de s'oublier lui-même sous la pression de ses accusateurs. C'est la chose la plus importante : il ne veut pas négliger sa pensée, ni s'oublier comme sujet. Ce mot oublie est présent tout au début de l'Apologie :

Je ne sais, Athéniens, quelle impression mes accusateurs ont faite sur vous. Pour moi, en les entendant, peu s'en est fallu que je ne me méconnusse moi-même [c'est-à-dire, que je m'oubliasse moi-même], tant ils ont parlé d'une manière persuasive ; et cependant, à parler franchement, [...] ils n'ont pas dit un mot qui soit véritable ; et de ma bouche vous entendrez la vérité tout entière.

Que signifie donc oublier?

Foucault, dans Le courage de la vérité, rappelle le passage de l'Apologie lorsque Chéréphon interpelle l'oracle de Delphes : «Quel est donc le Grec qui est plus savant de Socrate ? » et obtient en réponse : «Nul n'est plus savant que Socrate ${ }^{68}$. Mais Socrate ne se contente pas de ce prononcement de la Pythie et recherche lui-même la vérité du verdict. En premier, il discute

\footnotetext{
${ }^{66}$ M. FOUCAULT, Le courage de la vérité, Paris, Hautes Études, Gallimard-Seuil, 2009, p. 84.

${ }^{67}$ Ibid., p. 69.

${ }^{68}$ Ibid., p. 75.
} 
avec un homme politique et il comprend qu'il n'est pas plus sage que lui ; ensuite, il interroge des poètes qui expliquent leur activité comme due à une inspiration divine ; il parle encore avec les artisans qui, héritiers de connaissances spécifiques, prétendaient en savoir plus que lui. À la fin, Socrate comprend l'oracle : il est lui-même à la fois le plus ignorant et - pourtant, et par là même - le plus sage. Socrate voit qu'il a une mission envers ses concitoyens : il a été choisi par les Dieux pour les convaincre que la reconnaissance de leur ignorance peut être le premier pas sur la voie de la sagesse. Pour notre réflexion sur le temps, le plus important est que Socrate exclut de dépendre d'une prédiction future, d'un savoir qui est donné par l'extérieur (c'est le cas des sages qui se disent inspirés), ou d'un savoir déjà acquis (les artisans), ou de prospecter un futur (les politiciens). L'acte de Socrate est de refuser un savoir qui soit déterminé par le passé ou par l'avenir (l'oracle, que Socrate même soumet à examen). On est ici, désormais, dans une mémoire laïque. La sagesse est ce qu'on produit à l'instant, pendant le travail que l'on fait pour y être. Elle n'est pas in mente dei, ni dans une inspiration douteuse et incertaine, ni dans ce qui s'est déjà passé (l'expérience mécanique des artisans). La sagesse est l'activité du moment.

Socrate choisit de faire correspondre la pensée au moment même de son élaboration. C'est ce qu'explique Foucault :

Tous, que ce soit ces hommes d'État ignorants ou ces artisans savants, ont ceci en commun qu'ils croient connaître les choses qu'en réalité ils ne savent pas, alors que Socrate, lui, sait qu'il ne les sait pas. Il n'a sans doute pas le savoir de certains autres (les artisans), mais il n'a pas non plus leur ignorance. C'est cela (cette enquête, cette mise en question, ce questionnement, cet examen des autres en comparaison avec lui-même) que Socrate appelle, dans ce texte, l'exetasis. Exetazein, c'est : soumettre à l'examen ${ }^{69}$.

Soumettre à l'examen est l'ultime acte dialogique de Socrate lors qu'il raisonne avec Criton sur la positivité du choix de mourir.

La dernière phrase de Socrate dans le Phédon, interprétée différemment par plusieurs auteurs et à laquelle Dumézil donne une explication différente, c'est : «Criton, nous devons un coq à Asclépios ; payez-le, ne l'oubliez pas ». Une phrase que Lamartine, Nietzsche et d'autres auteurs interprètent comme un remerciement pour l'être en train d'être libéré de la vie. «O Criton, la vie

${ }^{69}$ M. FOUCAULT, Le courage de la vérité, op. cit., p. 77. 
est une maladie ${ }^{70}$, écrit Nietzsche dans Le gai savoir. Cependant, soutient Dumézil ${ }^{71}$, cet acte de reconnaissance est dû au fait que Socrate et Criton sont arrivés en pleine coopération, à travers l'échange de leurs convictions, à préférer la mort de Socrate à une vie de fugitif. Criton surmonte toute hésitation grâce au dialogue avec Socrate. On sait qu'il avait voulu persuader Socrate de se soustraire à la peine capitale pour garantir l'avenir de ses enfants, pour sauvegarder l'honorabilité de ses amis qui se préoccupaient pour lui, etc. Au fond, ils agissent pour être conformes, pour correspondre à l'opinion de la multitude, une idée que Socrate, à force d'argumenter, fait succomber définitivement. Socrate ne veut pas sortir (mot répété à maintes reprises), s'en aller pour sa satisfaction personnelle, pour se soulager de la vie; selon Sénèque, sa préoccupation n'est pas de mourir mais de soulager ses amis et en général les hommes de la crainte de mourir et de la prison ${ }^{72}$.

Le don du coq à Asclépios est donc dû, selon Dumézil, à ce parcours fait ensemble, bienveillant, qui les conduit aux mêmes conclusions. Un parcours aussi essentiel pour Socrate que pour Criton, grâce « à ce mélange d'amitié temporelle et d'amour philosophique dont parle le Phèdre, et qui rend indissociables les êtres qui en sont touchés $»^{73}$.

Dans le dialogue, Criton finit par accéder au choix de Socrate, le chemin a été fait ensemble (nous) ; Socrate ayant décidé que le moment de mourir est arrivé, ne resteront que les autres (vous). Il y a dans la structure de la phrase un monde qui se produit dans le hic et nunc. Il soude vie et mort dans le même moment, dans la même phrase. Tu, nous, vous, lorsque je ne serai plus

\footnotetext{
${ }^{70}$ F. NIETZSCHE, Le gai savoir - 'la gaya scienza', Textes et variantes établis par Giorgio COLLI et Mazzino MONTINARI, traduits de l'allemand par Pierre KLOSSOWSKI, éd. revue corrigée et argumentée par Marc B. de LAUNAY, Paris, Gallimard 1982, pp. 231-232. [1882-1887].

${ }^{71}$ Georges DUMÉZIL, "Le moyne noir en gris dedans Varennes » sotie Nostradamus suivie d'un " Divertissement sur les dernières paroles de Socrate », Paris Gallimard, 1984. Personne n'a relevé - écrit Foucault - que Dumézil a mis sous le même registre 'sotie' et 'divertissement' deux passages de l'histoire de notre culture. Dumézil utilise la même méthode pour une pensée considérée comme la plus irrationnelle dans notre culture occidentale, celle de Nostradamus, et pour la plus rationnelle, celle de Platon. M. FOUCAULT, Le courage de la vérité, op. cit., pp. 111-113.

72 SÉNÈQUE, Lettre 24, 4. «Socrate disserta dans sa prison ; il pouvait fuir, on lui offrait de le sauver, il ne le voulut pas et resta, pour ôter aux hommes leurs deux grandes terreurs, qui sont la mort et la prison ». Joseph BAILLARD (dir. et trad.), Euvres complètes de Sénèque le philosophe, Paris, Librairie L. Hachette, 1861. http://remacle.org/bloodwolf/philosophes/seneque/lucilius1.htm

${ }^{73}$ G. DUMÉZIL, «Le moyne noir en gris dedans Varennes » sotie Nostradamus suivie d'un 'Divertissement sur les dernières paroles de Socrate', op. cit., p. 168. Ce sont les réflexions d'un des personnages, Géphyrios, qui dialogue avec Kosshyphidios dans l'essai de Dumézil.
} 
là. Le je n'est pas prononcé dans cette dernière phrase de Socrate. Socrate peut donc s'oublier soi-même, en ayant partagé avec ses amis cette décision ultime.

\section{Théâtre et vie : le cynisme}

Seulement les personnes superficielles ne jugent pas des apparences. Le vrai mystère du monde est le visible, non l'invisible. Le portrait de Dorian Gray, Oscar Wilde ${ }^{74}$

Toute l'herméneutique du sujet est développée par Foucault autour de l'explicite. Il a employé à plusieurs reprises le mot théâtre, qui correspond bien à l'image d'un lieu où le vrai assume tout son poids en public.

Le théâtre est à l'origine de la culture grecque. John Burnet illustre comment les dialogues de Platon constituent une prose narrative réaliste, dans le sens où tous les intervenants ont un langage propre, se distinguent des autres, en étant aussi caractérisés, déjà personnages d'un drame. À la différence, par exemple, de Thucydide qui fait parler tous ses personnages dans le même style $^{75}$.

Le théâtre d'Euripide devient une référence dans les travaux foucaldiens des dernières années. Ce théâtre constitue pour Foucault le point de rencontre entre la politique et l'exercice de la vérité. Le paradigme du théâtre ancien est vu comme le laboratoire de multiples actes de vérité. Le théâtre est le lieu par excellence du visible ${ }^{76}$.

Selon Scarpat, on trouve le tout premier témoignage du terme parrhesia dans le fragment fr.226 DK. de Démocrite. Cette parole est présente aussi dans les œuvres d'Euripide et d'Aristophane. On pourrait affirmer que ce mot constitue un pivot de la tragédie attique. Parrhesia est presque un synonyme de 'théâtralité'.

Deux textes de Giuseppe Scarpat permettent de comprendre l'importance et l'évolution, les traductions dans le temps du mot parrhesia. Foucault connaissait la première publication de

\footnotetext{
${ }^{74}$ Oscar WILDE, Il ritratto di Dorian Gray, Firenze, Sansoni, 1965, p. 32. The Picture of Dorian Gray, 1890.

${ }^{75}$ John BURNET, «Introduction », in id. Phoedo, London, Oxford University Press, 1911, XXXI.

${ }^{76}$ Voir : A. SFORZINI, "Dramatiques de la vérité : la parrêsia à travers la tragédie attique », in Daniele LORENZINI, Ariane REVEL et Arianna SFORZINI dir., Michel Foucault : éthique et vérité 1980-1984, op. cit., p. 157.
} 
Scarpat $(1959)^{77}$. Parrhesia greca, parrhesia cristiana ${ }^{78}$ est sorti après la disparition de Foucault, et traite d'une manière exhaustive la naissance du mot et son passage du monde grec au monde chrétien. Le mot parrhesia (franc-parler) est apparu pour la première fois au sein du théâtre grec ${ }^{79}$. Deux mots ont précédé ce terme et en sont très proches : isonomie qui signifie égalité devant la loi et surtout devant le pouvoir, et iségorie, le droit de parole paritaire dans une assemblée. Le mot isonomie apparaît déjà dans un commentaire poétique de 510 av. J.C. L'iségorie correspond à la pratique de renvoyer les décisions à la collectivité (on l'a vu au point 4 de cet article). Ces deux mots faisaient partie du vocabulaire politique grec courant.

La troisième parole-clé est parrhesia : «la liberté du citoyen privé de dire ce qu'il croit ; comment il le croit, contre qui il croit ; il [le mot parrhesia] représente une conquête et un privilège de la démocratie athénienne, elle est un imprescriptible droit du citoyen et au début est synonyme de citoyenneté pleine $»^{80}$. Seul un aveu public rend évidente la vérité.

On trouve des anticipations conceptuelles du terme dans l'Iliade lorsque Achille dit : « Je hais à l'égal des portes des Enfers celui qui cache une chose dans son cœur et en dit une autre » (Iliade 9, 314) ${ }^{81}$. L'idée de simuler, de cacher intentionnellement la vérité est mal vue ; cependant, on sait que la ruse, la mètis, a été une composante de la culture grecque. Eric Dodds dans The Greeks and the irrational ${ }^{82}$, d'une part, et Marcel Detienne et Jean-Pierre Vernant dans Les

${ }^{77}$ Giuseppe SCARPAT, « Le traduzioni latine del termine parrhesia», in Atti del sodalizio glottologico milanese, v. XII, anno 1959, pp. 7-59. C'est Laura CREMONESI dans «Askêsis, êthos, parrêsia : pour une généalogie de l'attitude critique ", in Daniele LORENZINI, Ariane REVEL et Arianna SFORZINI dir., Michel Foucault : critique et vérité 1980-1984, qui affirme que Foucault connaissait ce travail de Scarpat. Voir p. 136, note 1.

${ }^{78}$ G. SCARPAT, Parrhesia greca, parrhesia cristiana, Brescia, Paideia edizioni, 2001.

79 "Le terme 'naît' au théâtre, et se retrouve pour la première fois chez Euripide et Aristophane », Arianna SFORZINI, «Dramatiques de la vérité : la parrêsia à travers la tragédie attique », in Daniele LORENZINI, Ariane REVEL et Arianna SFORZINI dir., Michel Foucault : critique et vérité 1980-1984, op. cit., p. 144.

${ }^{80}$ G. SCARPAT, Parrhesia greca, parrhesia cristiana, op. cit., p. 35. Notre traduction, pour ce passage et les suivants du même livre.

${ }^{81}$ Ibid., p. 37. Je reprends ici la traduction du grec en français de Pierre Louis Claude M. GIN, Paris, 1786 http://homere.iliadeodyssee.free.fr/traducteur/gin/gin.htm. Cf. sur ce thème aussi : Carlo DIANO, Forma ed evento: principi per un'interpretazione del mondo greco, Venezia, Neri Pozza, 1952. Achille et Ulysse sont les termes opposés d'une comparaison pour comprendre la valeur de la mètis.

${ }^{82}$ Eric Robertson DODDS, Les grecs et l'irrationnel, Paris, Flammarion, 1977 [Berkeley : 1951] ; et Marcel DETIENNE et Jean-Pierre VERNANT, les ruses de l'intelligence. La mètis des grecs, Paris, Flammarion, 1974. Collection dirigée par Fernand Braudel. Selon ces auteurs, la pensée grecque a établi une dichotomie radicale entre être et devenir, l'intelligible et le sensible, contrairement aux penseurs chinois ou indiens. À partir du $\mathrm{V}^{\mathrm{e}}$ siècle av. J. C., la mètis, cette forme d'intelligence, a été refoulée dans l'ombre. 
ruses de l'intelligence. La mètis des grecs, d'autre part, en expliquent l'importance. Malgré les études qui valorisent ce côté consubstantiel à la culture grecque qui trouve en Ulysse sa pleine expression, la philosophie occidentale a depuis toujours choisi, élu la voie royale de la rationalité. La parrhesia, le franc-parler, s'inscrit dans cette empreinte.

La question de savoir s'il faut donner une plus grande importance aux lois écrites ou au contraire à l'échange dialogique (Antigone, Agamemnon) pour accomplir cette rationalité, cette correspondance entre les réflexions et les comportements a été l'objet d'une discussion politicophilosophique pendant la période la plus stable de la démocratie athénienne. Dans le Sophiste de Platon le personnage de l'étranger dit à Timée «Eh bien, pensée et discours ne sont qu'une même chose ${ }^{83}$.

Ione, personnage principal de la tragédie homonyme d'Euripide, revendique le droit à la parrhesia, au franc-parler, parce qu'il est de mère athénienne. «En définitive c'est la première et vraie parrhesia : un privilège des citoyens libres de la démocratie athénienne dont sont privés les esclaves, les métèques, les étrangers ${ }^{84}$. Dans l'Hyppolite d'Euripide, Phèdre, avant de se suicider souhaite que ses enfants puissent avoir le droit à la parrhesia ${ }^{85}$.

Le mot est présent aussi dans des épitaphes d'importantes personnalités ${ }^{86}$.

Pourtant, la parrhesia devient à un certain moment une forme d'insulte, une manière incontrôlée de s'adresser à l'autre. Platon en avait condamné les excès, tout en appréciant la puissance du dire vrai.

Au fil du temps, le mot assume un caractère de plus en plus moral. Il l'avait été depuis le début, mais une fois « disparue la démocratie athénienne avec toutes ses libertés, Diogène devient le représentant de l'idéal de parrhesia, transposée de la polis au monde moral de la cosmopolis

\footnotetext{
${ }^{83}$ PLATON, «Sophiste» $263^{\mathrm{e}}$, in id. Sophiste, Politique, Philète, Timée, Critias, Édition établie par Émile CHAMBRY, Paris, Flammarion, (Garnier Frères, 1969), p.135. "Eh bien, pensée et discours ne sont qu'une même chose, sauf que le discours intérieur que l'âme tient en silence avec elle-même, a reçu le nom spécial de pensée ». Tout ce passage a été repris par Pavel A. FLORENSKIJ, Stupore e dialettica, Natalino VALENTINI dir., trad. du russe par Claudio ZONGHETTI, Lavis (Tn), Quodlibet, 2011, p. 50 [Dialektika, 1918-1922]. Hannah ARENDT en parle dans «Questions de philosophie morale », in id. Responsabilité et jugement, éd. établie et préfacée par Jérôme KOHN, trad. de l'anglais de Jean-Luc FIDEL, Paris, Payot, 2003, p. 119 [2003].

${ }^{84}$ G. SCARPAT, Parrhesia greca, parrhesia cristiana, op. cit., 42.

${ }^{85}$ M. FOUCAULT, « Theatrum philosophicum », Dits et écrits, II, op. cit., p. 99. Voir : G. SCARPAT, Parrhesia greca, parrhesia cristiana, op. cit., p. 40. (Euripide: Hyppolite 421-423).

${ }^{86}$ G. SCARPAT, Parrhesia greca, parrhesia cristiana, op. cit., pp. 53-54.
} 
hellénique $»^{87}$. Et «Diogène représente la personnification grecque de la liberté morale, la seule qui soit restée après la perte de la liberté politique $»^{88}$.

Cynisme et parrhesia constituent alors presque des synonymes. Les Cyniques manifestaient par ailleurs un refus absolu des cultes mystériques ${ }^{89}$.

Les Cyniques constituent une pierre de scandale, au moment où ils montrent publiquement ce qui était considéré comme privé, intime, et excèdent effrontément dans le franc-parler. Dans le fragment rappelé supra fr.226 D.-K. Démocrite écrit que «l'élément constitutif de la liberté est la parrhesia, danger en est la connaissance du kairos ${ }^{90}$. La franchise est la caractéristique d'un esprit libre ; mais il est difficile de déterminer le moment où il faut la montrer. En fait, peut gagner qui est plus malin, qui est plus rapide à saisir l'instant.

Kairos, c'est l'instant qu'il faut saisir ${ }^{91}$. Selon Plutarque, si on néglige ce moment, on rend vaine la parrhesia «la parrhesia est une médecine dont l'application exige toujours des conditions précises et des interventions à propos $»^{92}$. Le franc-parler provoque des réactions, fait courir des risques, se présente comme la parole dans son devenir par rapport aux autres qui l'écoutent.

Foucault destine la leçon du 8 février 1984 à l'examen de cette difficulté du franc-parler comme réelle expression de démocratie. On sait combien Socrate invite à négliger l'opinion de la multitude. En somme, en donnant à tout citoyen le droit de parole, la démocratie à Athènes, avait produit des déviations démagogiques. Démosthène dans la Troisième Philippique, Platon dans La République (Livre VIII, en 557b) et Aristote dans la Politique (Livre III, ch. 4, paragraphes 1276b-1277b) évoquent cette majorité qui fait valoir ses propres valeurs ou des intérêts pas vraiment nobles (je reprends ici les références que mentionne Foucault). Aristote s'interroge sur l'opposition entre meilleurs/moins bons et plus nombreux/moins nombreux qui font l'enjeu de la démocratie, qui ne constitue qu'un compromis. Foucault se demande : «Qu'est-ce qui fait qu'en démocratie, il y a une impuissance du discours vrai ? Est-ce l'impuissance du discours

\footnotetext{
${ }^{87}$ Ibid., p. 75-76.

${ }^{88}$ Ibid., p. 77.

${ }^{89}$ Ibid., p. 78.

${ }^{90}$ Ibid., p. 36.

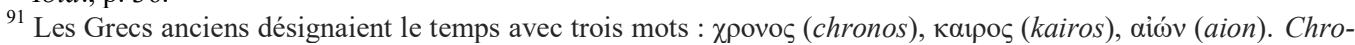
nos est le temps chronologique, quantitatif, séquentiel; Kairos est le moment à cueillir, l'opportunitas. Aion est le temps éternel, l'infini, «plus mince que tout instant », dit Foucault dans « Theatrum philosophicum », DE II, op. cit., p. 96.

${ }^{92}$ G. SCARPAT, Parrhesia greca, parrhesia cristiana, op. cit., p. 37.
} 
vrai en lui-même ? Certainement pas. [...] Elle est due à la structure même de la démocratie. [...] Parce qu'en démocratie on ne peut pas distinguer le bon et le mauvais orateur ${ }^{93}$.

Ce que Foucault dit et répète, c'est que la parrêsia exige une excellence morale. C'est donc l'ethos, la royauté de la vertu, qui qualifie la parrêsia ${ }^{94}$. Encore une fois, l'individualité prime sur une normalisation généralisée.

\section{La parrêsia chez Foucault}

On vient de le lire, la parrhesia est un des droits que la Phèdre d'Euripide souhaite pour ses enfants avant de se suicider. Elle devient un mot-clé. Si on rejette, en suivant Foucault, l'idée qu'on devrait chercher le côté obscur, caché et occulte, pour connaître et se connaître, seuls le visible, la franchise peuvent accomplir cette fonction essentielle dans un temps qui est le présent. Foucault aime croire que l'ethos d'un individu puisse correspondre totalement à son intimité. Même s'il n'ignore pas le risque de cette pratique. «Du moment qu'il y a parrêsia comme latitude pour tout le monde, il ne peut pas y avoir parrêsia comme courage de dire vrai. Et qu'en résulte-t-il ? Eh bien, dit Démosthène, il en résulte que, dans les assemblées, vous vous délectez à vous entendre flatter par des discours qui ne visent qu'à vous plaire ${ }^{95}$.

La parrêsia authentique devrait être une aléthurgie, une manifestation qui n'a rien de rhétorique, qui est prête à faire face aux réactions des autres à sa propre parole. Elle se produit et se donne à voir en même temps. Et en-dehors de toute métaphore, ce n'est certes pas par hasard que le mot apparaît pour la première fois sur les tréteaux.

Lorsque Foucault affirme que la parrêsia relève d'une dramatique du dire-vrai, il nous dit quelque chose de très important. Il envisage une expression de vérité qui engage la subjectivité éthique du locuteur: une pratique plutôt qu'une théorie, un style d'existence plutôt qu'un ensemble de critères formels, une dynamique de résistance critique plutôt que la formulation

\footnotetext{
${ }^{93}$ M. FOUCAULT, Le courage de la vérité, Leçon du 8 février 1984, op. cit., p. 40.

${ }^{94}$ Ibid., p. 51. Sur le concept d'éthos, Barthes écrit : «C'est l'affiche d'une franchise qui ne craint pas des conséquences et s'exprime à l'aide de propos directs, empreints d'une loyauté théâtrale ", Roland BARTHES, «L'Ancienne Rhétorique, aide-mémoire », in Communications 16, 1970, p. 211.

${ }^{95}$ Ibid., p. 39.
} 
de normes légitimées de véridiction. Un théâtre de la vérité donc, en contraposition aux Idées éternelles de la vérité-adéquation ${ }^{96}$.

Comme le dit Foucault «Coextensivité du souci de soi à l'art de vivre [...]. Le souci de soi devient coextensif à la vie $\gg{ }^{97}$.

S'il faut toujours interpréter critiquement l'œuvre de Foucault - non seulement parce qu'elle n'est pas monolithique mais encore en raison des contradictions qui peuvent s'y lover -, sa pensée réitérée, insistante au sujet de la parrêsia sonne comme un leitmotiv : défendre avec force, à visage ouvert, la liberté de penser dans une actualité publique et courageuse.

En analysant les multiples facettes de concepts qui nous accompagnent depuis l'antiquité sur l'interprétation du 'je', sur les conditions pour exercer le courage de la vérité, sur la parrêsia, Foucault tisse un fil rouge qui relie la pensée gréco-latine aujourd'hui, en indiquant des voies possibles, qui chaque individu en s'inscrivant en ce sillon pourrait parcourir.

\footnotetext{
${ }^{96}$ A. SFORZINI, « Dramatiques de la vérité : la parrêsia à travers la tragédie attique », in Daniele LORENZINI, Ariane REVEL et Arianna SFORZINI dir., Michel Foucault : éthique et vérité 1980-1984, op. cit., p. 148.

${ }^{97}$ M. FOUCAULT, L'herméneutique du sujet, Cours au Collège de France 1981-1982, op. cit., p. 84.
} 


\section{Déjà parus}

n. 1 : La documentazione autobiografica come patrimonio culturale : le testimonianze nella genesi del tempo, 2008.

n. 2 : Testimonianze, racconti di sé, memorie : il non-detto e il lavoro dell'interprete, 2009.

n. 3 : Le récit de soi : écouter, entendre, oü̈r, oreiller / L'ascoltare, il sentito dire, la phonè, in filigrana nei racconti di sé, 2010.

n. 4 : Fatum et téléologie dans le récit de soi / Tessere i racconti del sé tra fato e teleologia, 2011.

n. 5 : La figure du rebelle : écriture de soi et formes d'autolégitimation / Il ribelle, la scrittura di sé e forme di autolegittimazione / The rebel's self-legitimation, 2012.

n. 6 : L'ethos, mémoire autobiographique de l'homme de science / L'ethos, memoria autobiografica dell'uomo di scienza / The ethos, the scientist's autobiographical memory, 2013.

n. 7 : Raconter la science : autobiographies et biographies, de scientifiques entre hasard et nécessité/Raccontare la scienza : biografie a autobiografie di scienziati fra fato e necessità / Narrating science : biographies and autobiographies of scientists between chance and necessity, 2014.

n. 8 : Autobiografie e biografie sull'adozione / Autobiographies et biographies sur l'adoption / Autobiographies and biographies about adoption, 2015.

n. 9 : La naissance des idées dans des récits auto /biographiques / The origin of Ideas into the Autobiographical and Biographical narrations / La nascita delle idee nei racconti auto/biografici, 2016.

Mnemosyne, o la costruzione del senso est une revue indexée par ERIH PLUS, Europen Reference Index for the Humanities and social science

https://dbh.nsd.uib.no/publiseringskanaler/erihplus/periodical/info?id=488665

Mnemosyne, o la costruzione del senso, is a journal approuved from ERIH PLUS Europen Reference Index for the Humanities and social science.

Mnemosyne, o la costruzione del senso è una rivista indicizzata da ERIH PLUS, Europen Reference Index for the Humanities and social science. 